\title{
Metodologia de Aprendizagem por Riscos: Uma SIMULAÇÃO PARA PROJETOS DE INOVAÇÃO
}

\section{Gerson Pech}

Doutor em Física pelo Centro Brasileiro de Pesquisas Físicas/CNPq. Professor Associado da Universidade do Estado do Rio de Janeiro (UERJ) e professor do MBA em Gestão de Projetos da FGV. Rio de Janeiro, RJ, Brasil. E-mail: gerson@pech.com.br

\section{Lorena Costa do Espírito Santo}

Pós-graduanda em Sistemas e Estratégias da Inovação pelo Centro de Desenvolvimento Pessoal e Empresarial (CEDEPE). Analista de Projetos de Inovação do Instituto Euvaldo Lodi - IEL/PE. Recife, PE, Brasil. E-mail: lorecultura@hotmail.com

\section{Resumo}

Neste trabalho analisou-se a necessidade de gerenciar riscos em projetos de inovação utilizando os dados da Pesquisa Industrial de Inovação Tecnológica (PINTEC). O objetivo deste trabalho é propor uma metodologia de gestão de riscos adequada a projetos de inovação cujo pilar é o estudo dos riscos a partir das suas causas relevantes. A proposição tem a finalidade de gerar uma ferramenta de análise e de controle dos riscos para ser utilizada ao longo das diversas fases da inovação. Realizou-se uma simulação da aplicação dessa metodologia no projeto Núcleo de Gestão da Inovação (NUGI) do Instituto Euvaldo Lodi de Pernambuco (IEL PE) - instituição voltada para o apoio a projetos de inovação, entre outras atividades. Dessa forma, foi possível comprovar que o método desenvolvido é capaz de instrumentalizar o processo de aprendizagem dos riscos e de aumentar as chances de concretização das oportunidades.

Palavras-chave: Gestão de Riscos. Gestão da Inovação. Aprendizagem Organizacional.

\section{Abstract}

In this paper we analyze the need to manage the risks in innovation projects using data from the Industrial Research on Technological Innovation (PINTEC). The aim of this work is to propose a management methodology proper risk innovation projects whose pillar is the study of risks from their relevant causes. Our proposal aims to generate an analysis and control tool of risks to be use during the various phases of innovation. We conducted a simulation of the application of this methodology in NUGI (Innovation Management Core) project of Euvaldo Lodi Institute of Pernambuco state (IEL PE) - institution focused on the support of innovation projects, among another activities. Thus, it was possible to prove that the developed method is capable of exploiting the learning process of the risks and increase the chances of realization of opportunities.

Keywords: Risk Management. Innovation Management. Organizational Learning. 


\section{INTRODUÇÃO}

As razões da atual estagnação da taxa de inovação das empresas brasileiras, evidenciada pelas pesquisas que vêm sendo realizadas nos últimos anos (NEGRI, 2012; NEGRI; CAVALCANTE, 2013), precisam ser examinadas. Além das questões financeiras óbvias, como financiamento inadequado e necessidade de constantes investimentos, os motivos também podem estar associados, como se verá, à falta de cultura organizacional e à ausência de metodologias para gerenciar os riscos em atividades dessa natureza.

Projetos de inovação são geralmente complexos, não lineares e envolvem uma intricada composição de riscos e incertezas (GARUD; GEHMAN; KUMARASWAMY, 2011; STEFANOVITZ; NAGANO, 2014). Por isso, sem uma gestão eficaz de riscos perdem rapidamente o controle, consomem recursos incalculáveis e não chegam a lugar algum. De fato, práticas adequadas de gerenciamento de riscos têm forte relação com o desempenho de projetos (CARVALHO; RABECHINI, 2015; ZWIKAEL; AHN, 2011), ainda mais quando são projetos de inovação (KEIZER; HALMAN; SONG, 2002; O'CONNOR; RAVICHANDRAN; ROBESON, 2008). Entretanto, modelos rígidos e padronizados de Gestão de Riscos (GRIS) podem não ser apropriados para projetos de inovação. Ou seja, para projetos em que o diferencial é a criação e a pesquisa, metodologias tradicionais não são suficientes (ROVAI; CATTINI; PLONSKI, 2013). Isso acontece porque em várias organizações os processos de mitigação são bastantes conservadores e acabam impedindo ações só por não terem sido completamente testadas e estabelecidas.

Em termos corporativos, como apontam Nagano, Stefanovitz e Vick (2014), não bastam processos estruturados para que a inovação ocorra, pois é necessário um contexto que promova essa espécie de ação. E esse contexto passa por uma GRIS apropriada. Por exemplo, Garud, Gehman e Kumaraswamy (2011) verificaram que práticas que encorajam a iniciativa dos colaboradores não podem ficar de fora em projetos de inovação.

Assim, para entender o papel que os riscos desempenham nas empresas inovadoras brasileiras, foram analisados, neste trabalho, os dados das cinco rodadas da Pesquisa Industrial de Inovação Tecnológica (PINTEC) (INSTITUTO BRASILEIRO DE GEOGRAFIA E ESTATÍSTICA, 2002, 2005, 2007, 2010 e
2013). Fora as dificuldades de natureza estritamente financeiras (falta de recursos e de financiamento), a pesquisa revelou que Riscos Econômicos Elevados representam um grande obstáculo para o desenvolvimento de seus projetos de inovação. Por volta de $80 \%$ das empresas afirmam ter tido esse tipo de gargalo. Ou seja, a elaboração de um processo de gestão de risco aderente à inovação, para que as empresas tenham mais segurança no desenvolvimento das suas ideias, é certamente uma estratégia adequada.

Considerando esses indicativos, desenvolveu-se neste trabalho uma metodologia de GRIS em que a aprendizagem organizacional, nas diferentes fases dos projetos de inovação, é estruturada em uma Lista de Itens Relevantes (LIR). Seguindo a metodologia proposta, a LIR é utilizada para gerar a ferramenta de análise dos riscos do processo decisório.

Para averiguar se metodologias desse tipo têm sido desenvolvidas e estudadas atualmente, realizou-se um cruzamento na base SCOPUS para os últimos dez anos (2005 a 2015) usando o termo "knowledge management" em conjunto com as palavras: risk e innovation. A pesquisa foi realizada para título, abstracte keyword. O resultado obtido identificou 49 trabalhos, sendo que apenas três deles tratam especificamente de metodologias de GRIS em projetos de inovação (ALHAWARIA, 2012; JACQUIER $\square R O U X ;$ PARAPONARIS, 2012; SPERO et al., 2012). A maior parte dos outros papers resultante da busca já mencionada, ou objetiva analisar os riscos da implementação de um processo de distribuição de conhecimento, ou trata da integração entre Gestão do Conhecimento e GRIS em projetos que não são de inovação.

Nesse sentido, este trabalho preenche uma lacuna da literatura apresentando uma forma de alinhar o tratamento dos riscos da inovação à gestão do conhecimento corporativo.

Como resultado dessa metodologia, o conhecimento existente a respeito do projeto pode ser sistematizado. Foram reduzidos os riscos à medida que se gerou e agregou conhecimento, na medida certa, para o desenvolvimento da inovação (CUI; CHAN; CALANTONE, 2014). Seguindo argumento similar, Rice, O'Connor e Pierantozzi (2008) concluíram que práticas de GRIS orientadas à aprendizagem facilitam o sucesso, mesmo quando se trata de inovações radicais. 
Para investigar a aplicação da metodologia, uma equipe de analistas de inovação do Instituto Euvaldo Lodi de Pernambuco (IEL PE), com a orientação dos autores deste trabalho, construiu, a título de simulação, as ferramentas de análise dos riscos para os projetos das empresas do Núcleo de Gestão da Inovação NUGI que o IEL PE apoia. A aplicação da metodologia teve o propósito específico de identificar as categorias de riscos e suas causas fundamentais para os projetos ligados ao NUGI.

Dessa forma, foi possível concluir que a metodologia desenvolvida tem os seguintes benefícios: (i) produz uma ferramenta para se conhecer o nível de risco a que o projeto está submetido; (ii) instrumentaliza o processo de aprendizagem $e$ a gestão do conhecimento na empresa inovadora; (iii) indica o que precisa ser executado para que as oportunidades sejam alavancadas; (iv) aumenta as chances reais de sucesso da inovação.

Este paper está estruturado da seguinte forma: na seção 2 expõe-se a base teórica do trabalho, que é composta pelas pesquisas sobre inovação realizadas pelo IBGE, das características principais dos processos de inovação e dos modelos descritos pela literatura de integração entre riscos e inovação; na seção 3 descreve-se a metodologia de GRIS desenvolvida por este trabalho; na seção 4 apresenta-se os resultados obtidos pela simulação da aplicação da metodologia no projeto NUGI; e, finalmente, na seção 5 encontra-se as conclusões e perspectivas.

\section{Base Conceitual}

Como base teórica para o desenvolvimento da metodologia proposta por este trabalho, apresentamos nesta seção, dados e informações referentes ao papel da GRIS em projetos de inovação. A primeira subseção revela dados de pesquisas realizadas pelo IBGE, enquanto as duas últimas mostram os conceitos predominantes que têm sido aplicados na área.

\subsection{Inovação e Riscos nas Empresas Nacionais}

Para conhecer o comportamento da inovação no Brasil é preciso analisar os dados nacionais da Pesquisa
Industrial de Inovação Tecnológica - PINTEC (IBGE, 2002, 2005, 2007, 2010 e 2013). Até agora são cinco edições, incluindo informações de 1998 a 2011. A PINTEC segue o Manual de Oslo (Organização para a Cooperação e Desenvolvimento Econômico, 2005) e suas informações referem-se a inovações de Produto e Processo (PP), organizacionais e de marketing. Metodologicamente, o trabalho apoia-se na coleta, sistematização e análise de um abrangente conjunto de estatísticas descritivas dos indicadores de inovação (IBGE, 2013).

Diversas pesquisas nacionais usam os dados da PINTEC (KATO et al., 2008; LAZZAROTTI; MARCON; BANDEIRA-DE-MELLO, 2014; LOPES; BARBOSA, 2014; SANTOS et al., 2014) o que mostra ser esse um retrato expressivo das inovações produzidas pelas empresas brasileiras. De fato, uma análise detalhada dos dados da PINTEC é capaz de revelar relacionamentos entre as variáveis pesquisadas e ações que devem ser empreendidas para aprimorar a inovação nacional. No nosso caso, procurou-se inferir o papel da GRIS no processo de inovação e sua importância para as organizações inovadoras.

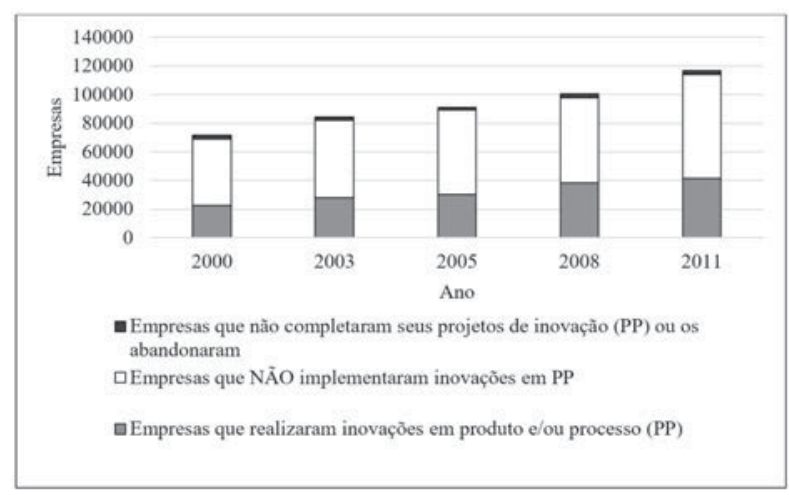

Figura 1: Empresas com potencial de inovação em PP Fonte: Adaptada de IBGE (2002, 2005, 2007, 2010 e 2013)

Primeiramente, é possível examinar a evolução do potencial inovador nacional desde 1998, primeiro ano da PINTEC. O histograma da Figura 1 representa essa evolução para os casos de inovações PP industriais. A quantidade de empresas com potencial de inovação é representada pela barra total. Em cada barra, está distribuída a quantidade de empresas que realizaram projetos de inovação $e$ a quantidade de empresas que não realizaram. A Figura 1 apresenta, também, o montante de empresas que começaram, 
mas não terminaram o projeto de inovação. A quantidade de empresas dotadas do potencial inovador cresceu $62 \%$ nesse período, passando de 72.000 para 116.660 (Figura 1). Entretanto, para entender se esse crescimento foi eficaz, é preciso investigar também a quantidade de empresas que desenvolveu projetos de inovação. Isso pode ser avaliado através da taxa de inovação (razão entre a quantidade de empresas que realizaram inovações e o total de empresas potenciais). No caso da indústria nacional, em 2000, a taxa de inovação era de $31,52 \%$, cresceu paulatinamente até 2005 passando para 33,27\% e em 2008 alcançou o índice de $38,11 \%$, ano em que o país registrou no primeiro trimestre uma expansão de $6,4 \%$ em relação ao mesmo período do ano anterior. No triênio seguinte, a taxa caiu para $35,56 \%$.

De forma geral, considerando todo o período, constatou-se que apesar de o número de empresas com potencial inovador ter aumentado, a taxa de inovação da indústria do país está praticamente estagnada. As causas dessa paralisia necessitam ser estudadas e, para isso, a própria PINTEC nos fornece alguns dados, como se verá a seguir.

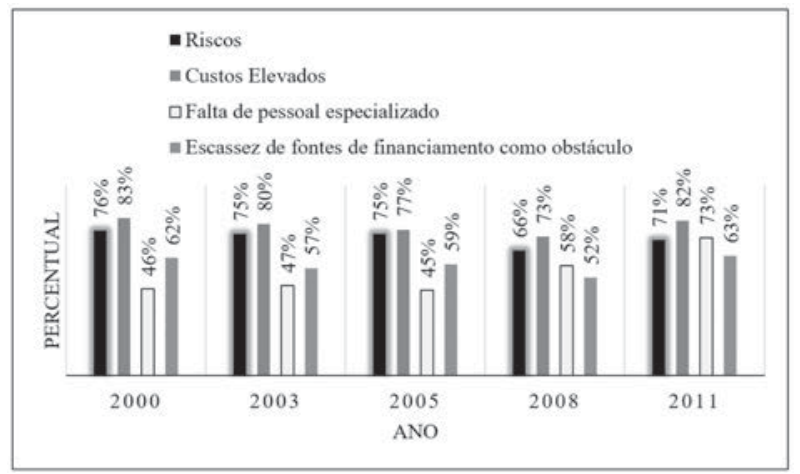

Figura 2: Obstáculos apontados pelas empresas que inovaram Fonte: Adaptada de IBGE (2002, 2005, 2007, 2010 e 2013)

A Figura 2 revela os principais obstáculos apontados pelas empresas que inovaram. São eles: Riscos econômicos elevados, Custos elevados, Falta de pessoal especializado e Escassez de fontes de financiamento. É relativamente grande a participação do item relacionado a riscos. Mais que $70 \%$ das empresas que realizaram projetos de inovação PP entendem Riscos econômicos elevados como sendo obstáculo. Ou seja, os riscos têm importância considerável na inovação $e$, por isso, precisam ser sistematicamente gerenciados.
Jacoski et al. (2014) obtiveram resultado análogo quanto à importância dos riscos. Eles avaliaram o nível de inovação tecnológica de 54 indústrias de Santa Catarina e mostraram que, também nesse caso, os riscos foram apontados como fator crítico dificultando as atividades de inovação.

A PINTEC também investigou as inovações organizacionais. Diferentemente das inovações em processos, que trata dos processos para fabricação de produtos, as organizacionais estão relacionadas à implantação de melhores práticas, procedimentos otimizados e técnicas de gestão. Assim, empresas que não foram inovadoras PP podem ter inovado em técnicas organizacionais, e vice-versa. Mas, será que existe dependência entre esses dois tipos de inovações?

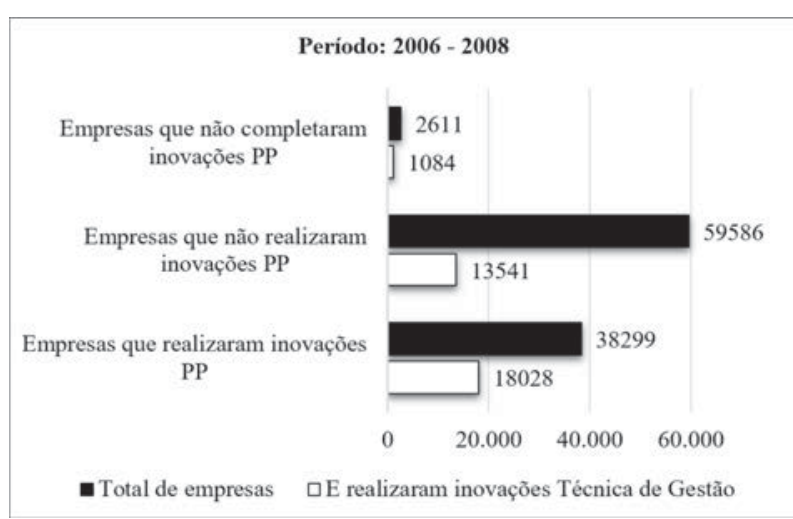

Figura 3: Inovação em Técnica de Gestão 2006 - 2008 Fonte: Adaptada de IBGE (2010)

A fim de examinar essa questão, apresenta-se na Figura 3 o quantitativo de empresas que desenvolveram inovações em Técnica de Gestão agrupadas de acordo com a mesma segmentação usada na Figura 1: (i) empresas que não completaram seu projeto de inovação PP; (ii) empresas que não realizaram projetos de inovação PP, (iii) empresas que realizaram inovações PP. Como mostra a Figura 3, de 2006 a 2008, das 59.586 empresas que não realizaram inovações PP, apenas 13.541 realizaram inovações em Técnica de Gestão (22,7\%), e das 38.299 que realizaram inovações $\mathrm{PP}, 18.028$ também realizaram inovações em Técnica de Gestão (47,1\%). Ou seja, o percentual mais que duplica em favor das empresas que realizaram os dois tipos de inovações.

Similarmente, para o período de 2009 a 2011 (IBGE, 2013), das 72.418 empresas que não realizaram inovações PP, apenas 20.139 realizaram inovações 
em Técnica de Gestão (27,8\%), e das 41.470 que realizaram inovações $\mathrm{PP}, 23.485$ também realizaram inovações em Técnica de Gestão (56,6\%). A taxa das que realizaram os dois tipos aumentou quase 10 pontos percentuais, considerando os dois períodos analisados. Fora isso, nesse último período, a taxa das que realizaram os dois tipos é o dobro das que apenas implantaram novas técnicas de gestão.

De fato, combinar inovações PP com inovações organizacionais pode ser uma perspectiva para melhorar o desempenho da empresa. Elas são diretamente dependentes, como apontam os dados das duas últimas rodadas da PINTEC. Além disso, considerando que um dos gargalos para a inovação é a dificuldade de gerenciar riscos, concluiu-se que é necessário um processo integrado - Riscos e Inovação - para aumentar a chance de sucesso dos projetos de inovação.

\subsection{Gestão da Inovação: estágios e portões de decisão}

Apesar de o processo de inovação ser não linear, ele passa, necessariamente, por estágios característicos. O início se dá através de etapas voltadas à geração de ideias e, na sequência, as fases vão ganhando atributos quantitativos. O caminho é percorrido no sentido da redução de incertezas de natureza técnica, de mercado, organizacionais e de recursos (SILVA; BAGNO; SALERNO, 2014). Diferentes estruturas foram concebidas para organizar esse complexo processo, cada qual apropriada para um tipo de inovação. Salerno et al. (2015) investigaram 132 projetos de inovação, e concluíram que em inovações incrementais as estruturas tradicionais são as mais utilizadas, enquanto o emprego de outros processos é típico de inovações de ruptura. Define-se o processo tradicional como composto de fases do tipo: prospecção de ideias, plano de negócio, projeto, desenvolvimento, testes e produção. De fato, não se pode definir uma estrutura que combine com todos os tipos de projetos e de cultura organizacional.

Um modelo bastante utilizado é o Stage-Gate ${ }^{\circledR}$ (COOPER, 1990) e suas variações (COOPER, 2008, 2009, 2010; ETTLIE; ELSENBACH, 2007). Trata-se de um roteiro conceitual que guia o projeto de inovação até o seu lançamento. A Figura 4 apresenta esse processo composto de cinco estágios e cinco portões, que são marcos de decisão (Go/Kill).

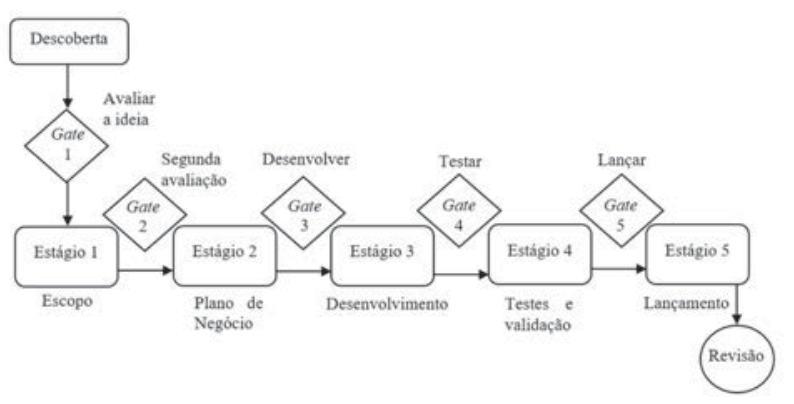

Figura 4: Stage-Gate ${ }^{\circledast}$ - um processo tradicional

Fonte: Adaptada de Cooper (2008)

Escolheu-se o Stage-Gate ${ }^{\circledR}$ para simular a MAR por ele suportar várias classes de projetos, quando adaptado. Contudo, outros modelos de Gestão da Inovação, compostos por fases, são igualmente adequados. Por exemplo, o modelo de Rozenfeld et al. (2006) possui estágios definidos com portões de decisão que vão desde a estratégia do negócio até a descontinuidade da operacionalização do produto, e poderia ter cumprido igual proposição. Outro modelo, proposto por Hansen e Birkinshaw (2007), teria aplicação similar ao do Stage-Gate ${ }^{\circledR}$, já que as duas estruturas de processo possuem características bem próximas. Hansen e Birkinshaw (2007) utilizam um processo denominado Cadeia de Valor da Inovação, possuindo apenas três estágios: geração da ideia, desenvolvimento e disseminação. Nesse caso, a MAR deveria ser aplicada nessas três fases do projeto. Seguindo a linha de representar a inovação por uma cadeia de valor, Srivastrava e Singh (2012) incorporaram a percepção de valor dos consumidores no processo de análise do desenvolvimento da inovação. Eles aplicaram um check list em cada fase do processo para compreender essa percepção.

De certa forma, a proposta deste trabalho é também de um check list, só que um check list de riscos orientado ao processo decisório. Efetivamente, a metodologia advogada por este paper é um exercício que visa à aprendizagem sobre as questões cruciais que afetam o projeto fase a fase. Por isso, vai de encontro à necessidade de avaliação e à imprescindível redução das incertezas, podendo ser utilizada independentemente da metodologia de Gestão da Inovação adotada pela empresa. 


\subsection{Integração entre Gestão de Riscos e Gestão da Inovação}

Para integrar GRIS e Gestão da Inovação deve-se levar em conta o caráter incerto de um projeto pioneiro, em que alto risco, em vez de ser algo ameaçador, pode ser, justamente, a razão para seguir em frente. Vários trabalhos (BOWERS; KHORAKIAN, 2014; HALMAN; KEIZER, 1994; OEHMEN et al., 2014; WANG; LIN; HUANG, 2010) foram desenvolvidos com o objetivo de estudar processos que conjuguem riscos e inovação. Bowers e Khorakian (2014) mostraram que existem fontes comuns de riscos para a maioria dos projetos de inovação. Dessa forma, a avaliação dessas fontes deve estar presente na estrutura do processo integrado. Os autores propõem um modelo de investigação no qual utilizam como fontes de riscos, os seguintes fatores: meio ambiente; técnico; recursos, gestão, marketing, integração e estratégia.

Segundo Wang, Lin e Huang (2010), é necessário que a GRIS da inovação esteja associada à estratégia da empresa, que não seja relativa a apenas um projeto específico. O objetivo é assegurar que o controle dos riscos seja incluído na estratégia de gestão de projetos da organização. Wang, Lin e Huang (2010) construíram uma estrutura de GRIS orientada ao desempenho usando os indicadores do BSC - financeiro, clientes, processos internos e aprendizagem - (KAPLAN; NORTON, 2005) e, assim, alinharam a GRIS da inovação à estratégia corporativa.

Também, com o propósito de integrar os dois processos, Mu, Peng e MacLachlan (2009) analisaram o poder que GRIS tem na melhora do desempenho de projetos de novos produtos. Seus testes foram realizados em empresas na China. Esse país tem sido palco de desenvolvimento de novos produtos a um baixo custo, logo é necessário entender como a GRIS tem sido aplicada por eles. Nesse caso, os autores utilizaram as seguintes categorias: tecnológico, organizacional e marketing. Eles construíram um modelo de análise dos riscos com base em: estudo com clientes e outros stakeholders; aquisição de conhecimento de fontes externas; e integrando internamente o conhecimento especializado (MU; PENG; MACLACHLAN, 2009). Ou seja, eles mostraram que a gestão eficaz dos riscos pressupõe o aumento do conhecimento em relação às suas fontes principais. Só dessa forma, é possível converter as ameaças da inovação em oportunidades reais.
A importância dos fatores utilizados por $\mathrm{Mu}$, Peng e MacLachlan (2009) já havia sido verificada no modelo de diagnóstico de riscos de Halman e Keizer (1994). Nesse caso, os autores apontam como parte vital do processo de identificação de riscos as lacunas de conhecimento tecnológico, organizacional e comerciais. Para avaliar essas lacunas, Halman e Keizer (1994) propõem a aplicação de questionários cujos resultados indicam o nível de risco para cada fator potencial. Dessa forma, a análise dos riscos não é, como no caso dos modelos convencionais, apenas um resultado direto da combinação entre a probabilidade de ocorrência e o impacto gerado. Mais do que isso, o nível de um risco é determinado pela habilidade ou inabilidade da empresa em influenciar uma situação. Esse aspecto será utilizado, também, no nosso modelo.

O modelo de Halman e Keizer (1994) foi inicialmente desenvolvido e utilizado na Philips Electronics, indústria multinacional de áudio, vídeo e iluminação. Desde então, o modelo foi aplicado em diversas indústrias, incluindo a Unilever (empresa que produz inúmeros bens de consumo em mais de 190 países) onde foi determinante no diagnóstico dos riscos, e na construção de uma base corporativa de conhecimento de riscos em projetos de inovação (KEIZER; HALMAN; SONG, 2002).

A avaliação dos riscos por meio de questionários, também, foi usada por outros autores. Por exemplo, Bowers e Khorakian (2014) apontam o checklist como adequado para a identificação de riscos em projetos de inovação. Segundo eles, essa técnica contribui para o aprendizado organizacional, disseminando o conhecimento na empresa.

Com o estudo apresentado, pode-se concluir que a GRIS em projetos de inovação deve prover uma estrutura preparada para considerar a alta quantidade de incertezas e o fluxo de informações requeridos para que sejam completados os estudos pertinentes, incluindo aí: a viabilidade tecnológica e financeira, o comportamento do mercado e dos competidores, todas as regulamentações, a demanda, a estratégia organizacional e demais fatores relacionados. Ou seja, a finalidade é prover aprendizagem das lacunas existentes nas áreas prioritárias do projeto, tais como: tecnologia, mercado, recursos, gestão, estratégia, marketing, organização, entre outras. 


\section{Metodologia de Aprendizagem por RIscos (MAR)}

O modelo proposto por este trabalho é fundamentado nos aspectos apresentados na seção 2 . Além disso, como observado por Alves Filho e Salm (2000), só a aprendizagem organizacional pode promover modificações profundas nos processos de gestão, transformando a empresa em uma organização que aprende continuamente. Ela é responsável pela formação das estratégias e por aprimorar o processo decisório das organizações (ALVES FILHO; SALM, 2000).

Assim, a MAR objetiva a criação de ferramentas de gestão dos riscos para o ciclo de vida do projeto. Não é uma técnica de utilização apenas para o início do desenvolvimento, nem apenas para a decisão sobre o lançamento. A necessidade de analisar os riscos e tomar decisões com base nessa análise é vital para o sucesso do negócio (OEHMEN et al., 2014). Dessa forma, a metodologia estabelece os processos para as empresas criarem uma ferramenta própria para a gestão dos riscos. Na seção 4 serão apresentados os resultados da MAR no projeto NUGI do IEL PE.

A metodologia foi elaborada para que a equipe e os gestores tomem conhecimento das características essenciais do projeto de inovação que estão desenvolvendo. Com efeito, a MAR pode contribuir para o processo de tomada de decisão que antecede cada estágio da inovação e pode ser utilizada, também, para determinar revisões das atividades ou mesmo alterações nas diretrizes de trabalho. Esses elementos foram mencionados nas seções 3 e 4 e fazem parte, também, do contexto de outros modelos (COOPER, 2008, 2009; SRIVASTRAVA; SINGH, 2012).

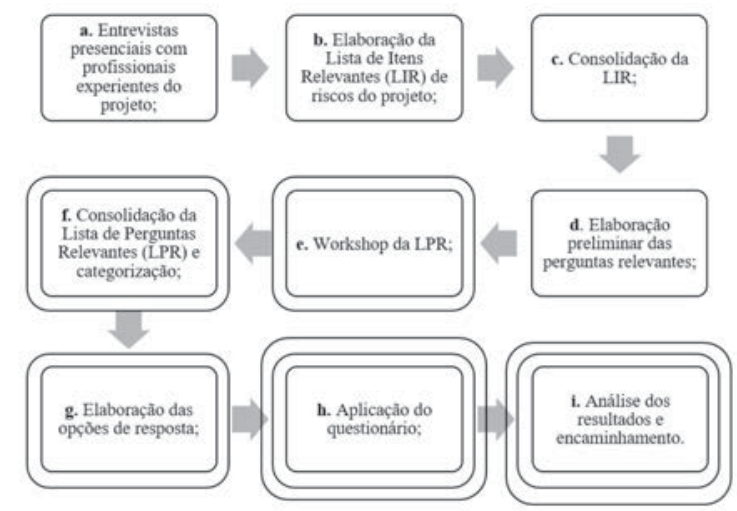

Figura 5: Diagrama da Metodologia de Aprendizagem por Riscos Fonte: Elaborada pelos autores deste artigo
Dividida em nove etapas, conforme diagrama da Figura 5, a MAR deve ser conduzida por um profissional externo e especializado em GRIS, que irá facilitar o processo, orientando a equipe na construção da ferramenta. O objeto final é a análise das respostas do questionário de riscos aplicado ao projeto de inovação em cada um dos seus estágios.

Fazem parte do processo as atividades de investigação (etapas $\mathbf{a}, \mathbf{b}$ e c c), a elaboração do questionário (etapas $\mathbf{d}, \mathbf{e}, \mathbf{f}$ e $\mathbf{g}$ ), sua aplicação (etapa $\mathbf{h}$ ) e a análise das respostas (etapa i). Entretanto, as nove etapas não devem ser empregadas a cada novo projeto. Para projetos similares, o início se dá na etapa $\mathbf{h}$, em que a ferramenta já está pronta para uso - processo destacado por três linhas na Figura 5. Para outros tipos de inovação, deve-se iniciar o processo na etapa e processo destacado por duas e três linhas na Figura 5. Com isso, é possível ganhar um processo flexível cujas ferramentas dependem da natureza do projeto. Segue o detalhamento de cada etapa.

a) Entrevistas presenciais com profissionais experientes do projeto: o processo começa com a identificação de pessoas que tem conhecimento e experiência na área. São realizadas entrevistas cujo objetivo é o levantamento das causas dos principais problemas que ameaçam o projeto. É preciso analisar os obstáculos ocorridos em outros projetos semelhantes e o que foi realizado para contornar a situação. Já nesta etapa, o coordenador do processo deve buscar as categorias principais dos riscos. De acordo com a literatura, os riscos da inovação devem estar relacionados a fontes econômicas, tecnológicas, de mercado, recursos, gestão, estratégia, marketing e organizacionais. Entretanto, cada projeto tem suas próprias fontes de riscos e, por isso, esse conjunto deve servir apenas como inspiração.

b) Elaboração da Lista de Itens Relevantes (LIR): o objetivo aqui é analisar o conteúdo das entrevistas e elaborar a LIR com base nas causas das dificuldades que foram apontadas. A LIR é o resultado de tudo o que foi discutido nas entrevistas e representa os principais fatores que impactam os objetivos do projeto. Vale frisar que a repetição desses fatores, em várias entrevistas, dá uma noção da relevância que eles têm para a organização. Porém, alguns fatores de baixa ocorrência, podem também 
ser relevantes, pois podem refletir riscos que a corporação, de forma silenciosa, não quer tratar. Esse é um ponto forte das metodologias que não usam apenas o brainstorming, porque ele pode nos remeter ao óbvio, ou a uma opinião já conhecida por toda a organização. Como exemplo, alguns dos itens da LIR, coletados das entrevistas no NUGI, são os seguintes: (i) Identificação do público-alvo; (ii) Demanda futura; e (iii) Elaboração da estimativa de custos.

c) Consolidação da LIR: o objetivo dessa etapa é expandir a abrangência do aprendizado sobre os riscos. Com as entrevistas presenciais - etapa anterior - deseja-se qualidade das respostas e, agora, virtualmente, se quer uma quantidade maior de profissionais interagindo para consolidar a lista. Portanto, o passo a passo dessa etapa é enviar a LIR para diversos profissionais envolvidos solicitando que eles confirmem os itens da lista e que identifiquem novos itens. A elaboração desse documento tem o propósito de fazer com que o conhecimento a respeito dos riscos seja utilizado, explicitamente, para garantir sucesso no desenvolvimento da inovação.

d) Elaboração preliminar da Lista de Perguntas Relevantes (LPR): a LPR deve ser um reflexo da LIR. Trata-se de converter cada um dos itens, ou grupo de itens, em perguntas. Isso é realizado com o intuito de investigar qual o conhecimento essencial, a respeito do item relevante, que é necessário para o pleno desenvolvimento do projeto de inovação, aquilo que ameaça os seus resultados. A pergunta é elaborada a fim de que sua resposta revele esse entendimento e que, com isso, seja possível estimar o risco correspondente. Ou seja, através das respostas será avaliada a lacuna de conhecimento de cada fator de risco. As perguntas associadas aos itens listados como exemplos na etapa b, são as seguintes: (i) O público-alvo foi identificado corretamente? (ii) Foi identificada a possibilidade de criação de novas demandas no decorrer do projeto? (iii) $\mathrm{O}$ plano de custos está completo considerando ambientes internos e externos e possíveis variações nas estimativas?

e) Workshop da LPR: nesta etapa a LPR passa por uma análise detalhada dos profissionais da empresa. Devem estar presentes representantes de diferentes áreas do projeto, o pessoal especializado e experiente em inovação, a área técnica, administrativa, jurídica, marketing, pro- dução etc. Evidentemente, a presença dos que participaram desde o início da construção do documento é imprescindível. A alta hierarquia também deve participar, pois o processo necessita de patrocínio político para alinhar-se com os procedimentos da organização. Nessa etapa, a LPR é validada e autorizada. Vale lembrar, que o documento gerado poderá ser utilizado em inúmeras outras inovações da organização, conforme exposto anteriormente. Nesse caso, esta etapa serve como revisão da LPR que já fora utilizada.

f) Consolidação da LPR e categorização: o resultado dessa etapa é a sistematização da GRIS em cada um dos estágios do processo de inovação. Após o workshop, atualiza-se a LPR em função das colaborações, consolidado o documento. É natural um rearranjo em termos de categorização a fim de atribuir a fonte de risco adequada a cada pergunta. É claro que a quantidade de perguntas depende da complexidade do projeto e do número de estágios do processo de inovação. Muitos estágios, cada um com muitas perguntas, tornará a metodologia inviável. Por outro lado, se a LPR for muito simples, a avaliação dos riscos será ineficiente, matando o processo. No caso em que as perguntas de uma categoria tenham impactos de magnitude significantemente diferentes no projeto, deve-se optar pela atribuição de pesos $\left(\alpha_{i}\right)$. Assim, cada pergunta terá um peso diferente na composição do risco da categoria.

g) Elaboração das opções de resposta: trata-se de formular quatro opções de respostas para cada questão. Cada uma das opções deve denotar um nível diferente de risco. Na verdade, uma escala qualitativa com parâmetros e critérios definidos. Ao fim do workshop (etapa e) deve-se selecionar internamente os profissionais que irão elaborar as opções de respostas, dividindo esse trabalho entre os participantes. Na criação das opções de resposta deve prevalecer a investigação do nível de conhecimento e do nível de incerteza em relação ao item relevante, pois o objetivo do processo é, conforme evidenciado nas seções anteriores, prover conhecimento das lacunas das fontes de riscos do projeto. Na seção 4 serão apresentados os resultados do estudo de caso deste trabalho.

h) Aplicação do questionário: os questionários devem ser aplicados ao final de cada estágio $e$ 
seus resultados deverão servir para o processo decisório. Sem isso, de nada valeu o levantamento das situações de risco e sua avaliação. Quem responde ao questionário deve ter isenção plena em relação aos resultados da investigação, mas total conhecimento da evolução do projeto de inovação. Ele pode ser um consultor, o próprio gerente do projeto, ou um membro da equipe capacitado para essa atividade. As respostas dos questionários são coletadas e contabilizadas revelando o nível de risco para cada categoria. Por exemplo, se uma das categorias é Mercado, o resultado será o grau de risco de Mercado para o projeto. $\mathrm{E}$, consequentemente, rastreando os resultados, toma-se conhecimento das fontes desses riscos e das ações que devem ser desempenhadas para reduzi-lo.

i) Análise dos resultados e encaminhamento: a aplicação da LPR terá como resultado o índice de risco $\left(R_{j}\right)$ para cada uma das categorias definidas (j). O valor de $R_{j}$ é determinado pela expressão: $R_{j}=\left(\sum_{1}^{N} r_{i} \cdot \alpha_{i}\right) / \sum_{1}^{N} \alpha_{i}$, onde $N$ é o número total de perguntas da categoria $j, r_{i}$ a resposta dada para a pergunta $i$ e $\alpha_{i}$ o seu peso. É possível analisar a evolução de $R j \mathrm{a}$ medida que o processo de desenvolvimento da inovação avança. Essa é uma forma de acompanhar o risco de uma categoria durante a execução do projeto. Note que as perguntas são distintas para cada um dos estágios. De fato, as avaliações nos estágios iniciais buscam aspectos qualitativos, $e$ à medida que o projeto progride as perguntas tornam-se mais detalhadas e objetivas. As perguntas e respostas foram cunhadas a partir daquilo que é relevante para o projeto, logo o resultado da avaliação irá indicar as lacunas de conhecimento existente. Assim, dependendo do resultado obtido pode-se optar por: (a) seguir o projeto de inovação; (b) parar o projeto; ou (c) voltar a um dos estágios anteriores.

A aprendizagem por riscos não acontece abruptamente na organização. A cada novo projeto em que a MAR é aplicada, o conhecimento sobre o significado do valor de Rj é obtido. Com isso, a organização é capaz de conhecer seu nível tolerável de risco, que viabilize o projeto, alavancando as oportunidades. Dessa forma, conhecer os riscos através da metodologia e poder controlá-los é a forma proposta por este trabalho para aumentar as chances de sucesso do projeto.

\section{Aplicação e Resultados da MAR}

\subsection{Considerações Iniciais da Aplicação da Metodologia}

A metodologia foi simulada no NUGI do IEL de Pernambuco durante o segundo semestre de 2014 e o início de 2015. Esse instituto é vinculado ao IEL Nacional e atua desde 1969 na integração das instituições de ensino e pesquisa com o setor produtivo do estado. O NUGI é um projeto de iniciativa da Confederação Nacional da Indústria (CNI) - e do Serviço Brasileiro de Apoio às Micro e Pequenas Empresas (SEBRAE), que tem o IEL PE como um dos responsáveis pela gestão e execução (MUNIZ, 2013). O objetivo do NUGI é promover a inovação nas empresas por meio do desenvolvimento de projetos que possam gerar aumento na sua competitividade.

A equipe de analistas de inovação que participou da aplicação da metodologia foi composta por seis profissionais de diferentes áreas, o que permitiu contribuições abrangentes para a LIR, para a LPR e para a escala de respostas. As áreas de formação $e$ pós-graduação dos analistas englobam: Comunicação, Marketing, Gerenciamento de Projetos, Economia, Engenharia de Produção, Gestão de Pessoas, Administração, Logística, Relações Públicas, Finanças e Controladoria. Eles possuem experiência de vai de dois a oito anos em projetos de inovação.

\subsection{Resultados e Discussões para a LIR}

Serão apresentados nesta seção os itens relevantes de risco, consolidando-se assim a LIR, conforme descrito na seção 3 . O processo de inovação foi orientado pelo Stage-Gate ${ }^{\circledast}$, com cinco portões, em que os riscos são avaliados. As categorias encontradas foram: Mercado \& Clientes; Financeiro \& Econômico e Estratégia \& Gestão. Segue abaixo a LIR categorizada.

Mercado \& Clientes - Estudo de mercado; Demanda futura; Aceitação dos serviços/produtos pelo público; Produto/serviço diferenciado; Posicionamento 
estratégico no mercado; Estudo para potencializar os aliados; Desenvolvimento de novas parcerias; Definição do público-alvo; Potencial de crescimento do projeto; Identificação de barreiras e restrições; Identificação adequada de parceiros; Identificação de novos e possíveis mercados potenciais; Empresas qualificadas para atender as demandas do projeto; Concorrentes com propostas similares para o mesmo público-alvo; Comprovação do diferencial competitivo; Recepção dos produtos/serviços pelo público-alvo; Entendimento quanto aos benefícios da inovação.

Financeiro \& Econômico - Desenvolvimento de parceria com o governo; Ampliação dos benefícios do projeto para o seu público-alvo; Possibilidade de aumento de receita; Aporte financeiro externo; Dimensionamento dos custos; Planejamento das aquisições; Estimativa da receita; Estudo de viabilidade econômico-financeira; Resultados financeiros.

Estratégia \& Gestão - Gestão do projeto; Fortalecimento das relações entre os membros da equipe; Implementação de uma nova cultura de inovação; Entendimento da equipe dos conceitos de inovação; Descrição das ações do projeto; Falta de indicadores de inovação; Metodologia adequada; Recursos materiais e humanos para desenvolver; Dimensionamento das estratégias de mobilização; Identificação dos stakeholders; Formulação do projeto e metas; Identificação da equipe técnica e gerencial; Habilidade para desenvolver o projeto; Empenho e comprometimento da equipe; Análise dos stakeholders; Aplicação de técnicas gerenciais.

Vale frisar, que a identificação dos itens relevantes não considerou um projeto específico de inovação, $e$ sim o conjunto de características dos vários projetos que têm sido apoiados pelos analistas do NUGI. O estudo dessas três categorias através dos itens relevantes tem os seguintes objetivos: (i) avaliar os riscos em cada etapa do processo de desenvolvimento da inovação; (ii) expor as lacunas de conhecimento de cada categoria; (iii) revelar o que ainda falta ser executado para se seguir adiante; (iv) ampliar a aprendizagem das questões-chave do projeto.

\subsection{Resultados e Discussões para a LPR}

Foram elaboradas 75 perguntas, distribuídas em cinco gates. Os riscos de cada gate são avaliados a partir de 15 perguntas, sendo cinco para cada uma das três categorias. Os analistas de inovação do IEL $\mathrm{PE}$, orientados pelos autores deste paper, elaboraram, também, as escalas de respostas, que são medidas por ri, cujo valor vai de 1 (baixo risco) a 4 (alto risco). É possível desconsiderar uma determinada pergunta, caso o avaliador entenda que ela não se aplica ao projeto. Nesse caso, ela não é incluída no cálculo de $R j$. Isso flexibiliza a ferramenta.

Os Quadros 1, 2 e 3 apresentam uma amostra das 75 perguntas. A seleção considerou a relevância das perguntas para o projeto. Essa amostra utilizou apenas os seguintes portões: Avaliar a ideia (Quadro 1) - que determina se a ideia deve seguir para o processo de inovação; Desenvolver (Quadro 2) - que avalia se a inovação deve entrar no processo de desenvolvimento; e Lançar (Quadro 3) - que analisa se a inovação deve ser lançada ao mercado. Esses portões foram escolhidos por serem comuns a vários projetos de inovação.

\section{Mercado \& Clientes}

\section{Foi realizado algum estudo junto a} possíveis clientes para entender e melhorar $r i$ a inovação a ser desenvolvida?

O estudo realizado trouxe contribuições relevantes para o desenvolvimento do produto/serviço, ajudando a entender a necessidade do mercado.

O estudo realizado trouxe contribuições para

o desenvolvimento do produto/serviço.

O estudo foi superficial e pouco conclusivo.

Não houve estudo.

Foi realizado algum estudo comparativo com outros projetos similares?

O estudo comparativo foi realizado com projetos similares internos, com ênfase no aproveitamento de pontos positivos para o desenvolvimento dessa inovação.

O estudo comparativo foi realizado com outros projetos similares, entretanto tais projetos são externos à organização.

\section{O estudo comparativo realizado com outros} projetos similares não apresentou relevância para o desenvolvimento da inovação.

Não foi realizado nenhum estudo comparativo.

FinANCEIRo \& Econômico

Os custos iniciais para o desenvolvimento da ideia foram estimados e existe disponibilidade? financeira?

Os custos iniciais foram estimados e se encontram dentro da disponibilidade financeira da empresa.
(2) 
Os custos iniciais foram estimados, contudo a empresa só pode cobri-los parcialmente.

Os custos iniciais foram estimados, contudo não estão dentro das possibilidades financeiras.

Não foi realizada uma estimativa de custos.

Os benefícios financeiros da inovação foram inicialmente estimados?

Foi verificado que a inovação é muito rentável, capaz de gerar benefícios financeiros consistentes.

Foi verificado que a inovação é razoavelmente rentável, capaz de gerar benefícios financeiros moderados para a empresa desenvolvedora.

Foi verificado que a inovação é pouco rentável, sendo apenas capaz de gerar benefícios financeiros equivalentes as despesas geradas, ou não foi realizado nenhuma estimativa para verificação dos benefícios financeiros.

Foi verificado que a inovação não é capaz de trazer benefícios financeiros para a empresa.

\section{Estratégia \& Gestão}

Foi realizada a análise das principais partes interessadas?

Foi realizada seguindo o procedimento consolidado na empresa para esse processo e apontou um forte comprometimento dos principais stakeholders.

Foi realizada e apontou certa possibilidade de comprometimento dos principais stakeholders.

A análise das partes interessadas ainda não foi realizada.

Foi realizada e apontou um alto grau de resistência em relação ao desenvolvimento do projeto.

A inovação foi alinhada à estratégia, missão e visão da empresa?

A inovação está alinhada à estratégia da empresa e visa contribuir para o fortalecimento da missão, visão e valores, bem como sua atuação no mercado.

A inovação está alinhada aos objetivos estratégicos da empresa, porém não contribuem para uma forte atuação no mercado.

A inovação tem um cunho estratégico, porém está em dissonância dos objetivos estratégicos da empresa, missão e visão.

Não, a inovação não está alinhada à estratégia da empresa, nem tão pouco contribuem para a missão e visão mesma.

Quadro 1: Lista de Perguntas Relevantes: Gate 1 (parte) Fonte: Elaborada pelos autores deste artigo.

Conforme indica o Quadro 1, na categoria Mercado \& Clientes, pode-se observar que a preocupação inicial é que sejam empreendidos estudos com os clien- tes para melhorar a concepção preliminar da inovação, assim como estudos comparativos com outros projetos. O conhecimento dos resultados dessas análises reduz os riscos iniciais, segundo este trabalho. Em relação à categoria Financeiro \& Econômico, a estimativa dos custos e benefícios no início do processo (Quadro 1), mesmo que grosseira, pois as informações ainda são incipientes, já revelam o perfil financeiro da inovação. Ignorar esse atributo do projeto é um indicativo de risco para o investimento. Já para a categoria Estratégia \& Gestão, este trabalho revela que é possível controlar melhor as ameaças do projeto nos estágios iniciais do processo, quando se entende as expectativas dos interessados e quando o projeto está alinhado com a política e as diretrizes da organização (Quadro 1).

\section{Mercado \& Clientes}

\section{As necessidades do público-alvo \\ foram identificadas, classificadas}

e relacionadas com custos?

ri

As necessidades dos clientes foram identificadas, classificadas e relacionadas ao custo do projeto.

Todo esse levantamento resultou em uma

melhor definição do produto/serviço.

As necessidades dos clientes foram identificadas e classificadas, contudo não houve um relacionamento com os custos, possibilitando apenas agregar qualidades técnicas do produto/serviço.

As necessidades dos clientes foram apenas identificadas, mas não foram classificadas e nem relacionadas com os custos do produto/serviço.

Assim, não será possível mensurar a relação entre a qualidade técnica e o custo dessa necessidade.

As necessidades do público-alvo não

foram identificadas e nem tratadas.

O público-alvo aprovou as características do produto ou serviço a ser lançado?

Foi realizada uma pesquisa de mercado que revelou um interesse absoluto pelas características e propriedades do novo produto/serviço.

Foi realizada uma pesquisa que revelou interesse por algumas características do novo produto/serviço.

O público-alvo desconhece todos os atributos do produto/serviço, sendo impossível sua aprovação ou não quanto às suas características.

A princípio, as características desta inovação não são aprovadas pelos possíveis clientes. 


\section{FinANCEIRO \& ECONÔMICO}

\section{Como foi elaborado o Estudo de Viabilidade \\ Econômico e Financeiro (EVEF) da}

ri inovação e qual o seu resultado?

Foi utilizada uma base de dados confiável para o EVEF e elaborada uma estimativa futura para o mercado levando em conta riscos e incertezas. Custos foram levantados diretamente com os fornecedores, fixos e variáveis. Foram analisados os principais indicadores econômicos (TIR, VPL e Payback). Todos eles altamente favoráveis.

Foi utilizada uma base de dados confiável para o EVEF, levando em consideração apenas os custos fixos e variáveis. Foram analisados os principais indicadores econômicos.

Foi utilizada uma base de dados confiável para o EVEF, levando em consideração apenas os custos.

As premissas para o projeto têm sofrido inúmeras alterações, impedindo resultados confiáveis.

Existem parcerias externas para este projeto, considerando o poder público e privado?

Já foram assinados contratos/convênios com organizações que costumam ser nossas parceiras detalhando como será realizado o aporte de recursos para a inovação.

A empresa faz parte de programa de incentivos do governo para desenvolvimento de projetos de inovação.

Foram identificadas algumas possibilidades de parcerias para este projeto.

Ainda não foram identificados parceiros para o projeto.

\section{Estratégia \& Gestão}

Qual o nível de capacitação da equipe técnica para desenvolver esse projeto?

A empresa tem histórico no desenvolvimento de projetos de inovação em diversos segmentos, bem como a estrutura física e técnica adequada para dar continuidade aos projetos.

A empresa vem buscando desenvolver projetos de inovação em gestão e tem equipe técnica capacitada para dar continuidade aos projetos.

A empresa nunca desenvolveu projetos de inovação antes, contudo possui um perfil inovador e é aberta a novas oportunidades que possibilitem o crescimento da empresa.

A empresa não tem potencial para o

desenvolvimento de propostas inovadoras.

Todos os planos do projeto foram acordados e validados pela alta gestão da empresa?

Através do processo já bem estruturado e várias vezes utilizado, os dirigentes participaram das reuniões de apresentação $e$ discussão do projeto $e$ validaram formalmente os documentos do plano.

Os dirigentes participaram apenas das reuniões finais para apresentação do plano de projeto e validaram formalmente seus principais documentos.
O plano ainda não foi validado pela

alta hierarquia da organização.

O plano não será validado pela alta hierarquia da organização.

Quadro 2: Lista de Perguntas Relevantes: Gate 3 (parte)

Fonte: Elaborada pelos autores deste artigo

O questionário referente ao gate Desenvolver é apresentado no Quadro 2. Pode-se ver pelas perguntas da categoria Mercado \& Clientes que aspectos quantitativos já ganham importância nesta etapa, relacionando necessidades do público com custos reais. Essa questão é taxativa, pois pode ser uma causa de estouro no orçamento, inviabilizando o projeto. Outra questão que essa mesma categoria aponta é que a pesquisa de mercado, aprovando as características prioritárias do produto, colabora para o entendimento do nível de risco. Em relação à categoria seguinte, é possível constatar pelo Quadro 2 que uma análise mais quantitativa da viabilidade econômica e financeira deve ser elaborada para poder examinar com detalhes os riscos associados. Também neste portão, busca-se entender qual a possibilidade de parcerias para o negócio, na perspectiva de compartilhar a oportunidade. Em termos de Estratégia \& Gestão, o nível de risco é menor para projetos que já possuem uma equipe técnica adequada e quando ele é aprovado pela alta hierarquia da empresa (Quadro 2).

\section{Mercado \& Clientes}

\section{O produto ou serviço tem sido constantemente} validado pelo seu público-alvo?

Existe um processo periódico para a validação pelos clientes que tem sido colocado em execução e cujos resultados são plenamente satisfatórios.

Os produtos e serviços foram validados pelos clientes em todas as etapas da construção do projeto.

Os clientes aprovaram o produto/serviço,

apenas, nas etapas iniciais do projeto.

Ainda não foi realizada nenhuma validação.

O produto desenvolvido é capaz de impor barreiras à entrada de competidores nesse mercado?

Ao que tudo indica não teremos

competidores por um bom tempo.

Para outras empresas desenvolverem um produto/serviço elas terão que adquirir conhecimentos que ainda não possuem.

O produto/serviço possui apenas barreiras

técnicas de baixo nível de complexidade. 
Já existem alguns possíveis competidores.

\section{FinANCEIRo \& ECONÔMICO}

As estimativas de custo para a produção/ operação foram alteradas?

Os custos planejados não foram alterados, estamos seguindo o planejamento financeiro.

Os custos planejados não foram alterados, contudo, para isso tivemos que fazer ajustes no produto/serviço.

Os custos sofreram alterações para que não houvesse mudança no escopo do produto.

Os custos, bem como o escopo do produto foram alterados.

\begin{tabular}{l|l|}
$\begin{array}{l}\text { As estimativas para a rentabilidade } \\
\text { do projeto foram alteradas? }\end{array}$ & ri \\
\hline $\begin{array}{l}\text { A previsão é de que a rentabilidade seja acrescida } \\
\text { devido a fatores positivos que vêm acontecendo e } \\
\text { que não estavam previstos nos estágios anteriores. }\end{array}$ & $(1)$ \\
\hline As estimativas não foram alteradas. & (2) \\
\hline A rentabilidade deverá ficar abaixo da esperada. & (3) \\
\hline É certo que a rentabilidade ficará abaixo da esperada. & $(4)$ \\
\hline
\end{tabular}

\section{EstratÉGIA \& GeStÃo}

A metodologia para desenvolvimento das ações de lançamento é adequada para atender e satisfazer o seu público-alvo?

Essa será, sem dúvida, a melhor forma de atingir o público-alvo do projeto.

Algumas ações já estão alinhadas de acordo com a expectativa da audiência do projeto.

Não se pode ainda entender qual a metodologia mais adequada para atender e satisfazer o público-alvo.

A metodologia precisará de mudanças, pois não é adequada.

A alta gestão está envolvida e comprometida no lançamento do projeto?

A alta gestão acompanhou e apoiou o projeto desde o início e participa, desde então, no engajamento de diversos stakeholders importantes.

A alta gestão acompanhou apenas algumas fases de desenvolvimento do projeto, porém oferece apoio e incentiva o engajamento de diversos stakeholders.

Parte importante da alta hierarquia da empresa tem se mostrado neutra.

Não há nenhum tipo de apoio da alta gestão da empresa para o desenvolvimento do projeto.

Quadro 3: Lista de Perguntas Relevantes: Gate 5 (parte) Fonte: Elaborada pelos autores deste artigo
Em relação ao portão de lançamento, o entendimento das barreiras existentes no mercado é peremptório, conforme sugerido na primeira categoria no Quadro 3. Em Financeiro \& Econômico, nosso trabalho mostra que a preocupação maior diz respeito às sucessivas alterações das estimativas de custos e rentabilidade, e o quanto isso pode ameaçar o projeto. De fato, um projeto de inovação precisa estar preparado para as sucessivas alterações que irão ocorrer e seus reflexos em prazo e custo. Nesse mesmo portão, como mostra o Quadro 3, os métodos que serão utilizados para a disseminação da inovação precisam ser adequados ao público-alvo, ou surgiram riscos elevados como resultado. Fora isso, neste momento, o apoio político dos dirigentes da organização é fator crítico de sucesso (Quadro 3).

\section{Conclusões e Perspectivas}

Riscos são constituintes indispensáveis da inovação. Logo, não existe Gestão da Inovação sem GRIS. Para entender qual a necessidade da integração entre esses dois processos nas indústrias nacionais, foram analisados, neste trabalho, os dados dos cinco ciclos da pesquisa de inovação - PINTEC - correspondendo ao período que vai de 1998 a 2011.

Com os dados da pesquisa concluiu-se que os riscos são um dos principais obstáculos em projetos de inovação, mesmo para as empresas que têm inovado. Fora isso, a pesquisa revela que existe uma forte correlação entre a taxa de inovação PP e a taxa de inovação organizacional. Considerando os dois últimos ciclos da PINTEC, aproximadamente $25 \%$ das empresas que não realizaram inovações PP, realizaram inovações em Técnica de Gestão. Enquanto, aproximadamente, $50 \%$ das que inovaram em PP, também inovaram em Técnica de Gestão. Com isso, concluiu-se o quanto é relevante inovar em técnicas de gestão para, também, inovar em produto e processo.

Dessa forma, desenvolveu-se, neste trabalho, uma técnica de GRIS adequada a projetos de inovação. O objetivo da técnica é prover conhecimento e aprendizagem das principais causas dos riscos envolvidos no projeto. Assim, é possível estudar as lacunas de conhecimento existentes no projeto considerando os seus fatores prioritários, como: tecnologia; mercado; 
clientes; e organização; entre outros. A partir desse método foi criada uma ferramenta de análise e controle dos riscos, aplicável independente do processo de Gestão de Inovação adotado.

Realizou-se uma simulação no Núcleo de Gestão da Inovação (NUGI) do Instituto Euvaldo Lodi de Pernambuco (IEL PE) que mostrou que os riscos dos projetos de inovação possuem causas associadas a Mercado \& Clientes, Financeiro \& Econômico e Estratégia \& Gestão. Partindo dessas categorias, foram construídas a LIR e a LPR, que constituem os objetivos específicos desse trabalho. Assim, nesse caso, constatou-se que:

a) Os riscos nas etapas iniciais terão níveis baixos se os estudos com os clientes ou analogias com projetos similares forem eficazes para aprimorar a inovação, se forem identificados benefícios financeiros no início do processo e quando procedimentos consolidados mostrarem existir compromisso dos principais stakeholders.

b) Os riscos nas etapas intermediárias diminuirão se o aprimoramento da inovação for um processo contínuo com participação dos clientes, se estiverem relacionadas a custos, se for elaborada uma estimativa futura levando-se em conta as incertezas; se já existirem contratos/ convênios firmados assegurando aporte financeiro para a inovação e se existir comprometimento da alta hierarquia nesta fase.

c) Os riscos para a etapa de lançamento serão reduzidos se os clientes validarem periodicamente o projeto; se não existir competidores; se o orçamento for seguido; se a metodologia para desenvolvimento das ações de lançamento for adequada para atender e satisfazer ao seu público-alvo e pelo engajamento da alta hierarquia da organização.

Dessa forma, concluiu-se que a metodologia desenvolvida tem os seguintes benefícios para as empresas inovadoras: (i) produz uma ferramenta contributiva para conhecer o nível de risco a que o projeto está submetido; (ii) instrumentaliza o processo de aprendizagem e gestão do conhecimento na empresa inovadora; (iii) indica o que precisa ser executado para que as oportunidades sejam alavancadas; e (iv) aumenta as chances reais de sucesso da inovação.

É importante ressaltar o caráter restrito da nossa aplicação, que não objetiva generalizar os fatores prioritários dos projetos de inovação, nem as suas questões relevantes. Os resultados aqui obtidos referem-se à aplicação da metodologia no campo da amostra especificada no paper.

A fim de dar continuidade ao trabalho, é preciso propor a aplicação da metodologia em projetos de inovação de diferentes áreas e naturezas, objetivando um estudo comparativo que indique outros benefícios que podem ser gerados para as empresas inovadoras. Por outro lado, um aprimoramento da metodologia poderia incluir funções de correlação entre os itens relevantes, associando-os de forma direta ou inversa a partir de suas características.

\section{ReferÊNCIAS}

ALHAWARIA, S. Knowledge-Based Risk Management framework for Information Technology project.

International Journal of Information Management, [S.l.], v. 32, n. 1, p. 50-65, feb. 2012. Disponível em: <http://dx.doi.org/10.1016/j.ijinfomgt.2011.07.002>. Acesso em: 7 out. 2015.

ALVES FILHO, A.; SALM, J. F. A formação da estratégia pela aprendizagem organizacional. Revista de Ciências da Administração, Florianópolis, ano 2, n. 3, p. 7-16, abr. 2000. Disponível em: <https://periodicos.ufsc.br/index.php/ adm/article/view/8032 > . Acesso em: 14 out. 2015.

Bowers, J.; KHORAKIAN, A. Integrating risk management in the innovation project. European Journal of Innovation Management, UK, v. 17, n. 1, p. 25-40, 2014. Disponível em: <http://dx.doi.org/10.1108/EJIM01-2013-0010>. Acesso em: 14 out. 2015.

CARVALHO, M. M. de; RABECHINI JUNIOR, R. Impact of risk management on project performance: The importance of soft skills. International Journal

of Production Research, UK, v. 53, n. 2, p. 321340, jan. 2015. Disponível em: <http://dx.doi. org/10.1080/00207543.2014 .919423 > . Acesso em: 14 out. 2015.

COOPER, R. G. Stage-gate systems: a new tool for managing new products. Business Horizons, USA, v. 33, n. 3, p. 44-54, may-jun. 1990. Disponível em: <http://econpapers.repec.org/article/ eeebushor/ v_3a33_3ay_3a1990_3ai_3a3_3ap_3a44-54.htm >. Acesso em: 14 out. 2015. 
COOPER, R. G. Perspective: The Stage-Gate Idea-to-Launch Process - update, what's new and NexGen Systems. The Journal of Product Innovation Management, USA, v. 25, n. 3, p. 213-232, may, 2008. Disponível em: <http:// dx.doi.org/10.1111/j.1540-5885.2008.00296.x>. Acesso em: 14 out. 2015.

COOPER, R. G. How companies are reinventing their idea-to-launch methodologies. Research Technology

Management, USA, v. 52, n. 2, p. 47-57, feb. 2009. Disponível em: <http://www. stage-gate.net/downloads/ wp/wp_38.pdf>. Acesso em: 14 out. 2015.

COOPER, R. G. The Stage-Gate idea to launch system. In: SHETH, J. N.; MALHOTRA, N. K. (Org.). Part 5: Product Innovation and Management, Wiley International Encyclopedia of Marketing. USA: John Wiley \& Sons, 2010.

CUI, A. S.; CHAN, K.; CALANTONE, R. The learning zone in new product development. IEEE Transactions on Engineering Management, USA, v. 61, n. 4, p. 690-701, nov. 2014. Disponível em: <http://dx.doi. org/10.1109/TEM.2014.2331757> . Acesso em: 14 out. 2015.

DE NEGRI, F. Elementos para a análise da baixa inovatividade brasileira e o papel das políticas públicas.

Revista USP, São Paulo, v. 93, p. 81-100, mar.-abr.maio, 2012. Disponível em: < http:/www.revistas.usp.br/ revusp/article/view/45004 > . Acesso em: 14 out. 2015.

DE NEGRI, F; CAVALCANTE, L. R. Análise dos dados da PINTEC 2011, Nota Técnica, n. 15. Brasília, DF: IPEA, dez. 2013. Disponível em: < http://www.ipea.gov. br/portal/images/stories/ PDFs/nota_tecnica/131206_ notatecnicadiset15.pdf > . Acesso em: 14 out. 2015.

ETTLIE, J. E.; ELSENBBACH, J. M. Modified Stage-gate: regimes in new product development. The Journal of Product Innovation Management, USA, v. 24, n. 1, p. 22-31, jan. 2007. Disponível em: <http://dx.doi.org/10.111 1/j.1540-5885.2006.00230.x>. Acesso em: 14 out. 2015.

GARUD, R.; GEHMAN, J.; KUMARASWAMY, A. Complexity arrangements for sustained innovation: lessons from 3M Corporation. Organization Studies, [S.l.], v. 32, n. 6, p. 737-767, jun. 2011. Disponível em: <http://dx.doi.org/10.1177/0170840611410810>. Acesso em: 14 out. 2015.
HALMAN, J. I. M.; KEIZER, A. Diagnosing risks in product-innovation projects, International Journal of Project Management, [S.1.], v. 12, n. 2, p. 75-80, may 1994. Disponível em: <http://dx.doi. org/10.1016/02637863(94)90013-2>. Acesso em: 14 out. 2015.

HANSEN, M. T.; BIRKINSHAW, J. The innovation value chain. Harvard Business Review, USA, v. 85, n. 6 , p. 121-130, jun. 2007. Disponível em: <https://hbr. org/2007/06/the-innovation-value-chain >. Acesso em: 14 out. 2015 .

\section{INSTITUTO BRASILEIRO DE GEOGRAFIA E} ESTATÍSTICA - IBGE. Pesquisa industrial de inovação tecnológica - PINTEC 2000. Rio de Janeiro: IBGE, 2002. Disponível em: <http://www.pintec.ibge.gov.br/ index.php?option $=$ com_content\&view $=$ article\&id $=45 \&$ It emid $=12>$. Acesso em: 14 out. 2015.

Pesquisa industrial de inovação tecnológica PINTEC 2003. Rio de Janeiro: IBGE, 2005.

Pesquisa de inovação tecnológica - PINTEC

2005. Rio de Janeiro: IBGE, 2007.

Pesquisa de inovação tecnológica - PINTEC

2008. Rio de Janeiro: IBGE, 2010.

Pesquisa de inovação - PINTEC 2011. Rio de Janeiro: IBGE, 2013.

JACOSKI, C. A. et al. Análise do desempenho da inovação regional - um estudo de caso na indústria.

Revista de Administração e Inovação, São Paulo, v. 11, n. 2, p. 71-88, abr.-jun. 2014. Disponível em: < http:// dx.doi.org/10.5773/rai.v11i2.1049>. Acesso em: 14 out. 2015.

JACQUIER-ROUX, V.; PARAPONARIS, C. Diversity and knowledge sharing: An analysis of integration processes in multinational firms (MNFs). VINE, [S.l.], v. 42, n. 3/4, p. 335-349, 2012. Disponível em: <http://dx.doi. org/10.1108/03055721211267477> . Acesso em: 7 out. 2015.

KAPLAN, R. S.; NORTON, D. P. The balanced scorecard: measures that drive performance. Harvard Business Review, USA, v. 83, n. 7, p. 172-180, jul. 2005. Disponível em: <https://hbr.org /2005/07/the-balancedscorecard-measures-that-drive-performance $>$. Acesso em: 14 out. 2015 . 
KATO, E. M. et al. Padrões de cooperação tecnológica entre setores na indústria brasileira: uma análise quantitativa dos dados da PINTEC 2001-2003. Revista de Administração e Inovação, São Paulo, v. 5, n. 3, p. 126-140, set.-dez. 2008. Disponível em: < http://dx.doi. org/10.5585/rai.v5i3 .253>. Acesso em: 14 out. 2015.

KEIZER, J. A.; HALMAN, J. I. M.; SONG, M. From experience: applying the risk diagnosing methodology.

The Journal of Product Innovation Management, USA, v. 19, n. 3, p. 213-232, may 2002. Disponível em: <http://dx.doi.org/10.1111/1540-5885.1930213>. Acesso em: 14 out. 2015.

LAZZAROTTI, F; MARCON, R.; BANDEIRA-DE-MELLO, R. Recursos para inovação e desempenho: uma análise da invariância de mensuração em firmas de setores de alta intensidade tecnológica, no Brasil. Revista de Administração e Inovação, São Paulo, v. 11, n. 4, p. 33-57, out.-dez. 2014. Disponível em: < http://dx.doi. org/10.5773/rai.v11i4.1007>. Acesso em: 14 out. 2015.

LOPES, D. P. T.; BARBOSA, A. C. Q. Management and organizational innovation in Brazil: evidence from technology innovation surveys. Production, São Paulo, v. 24, n. 4, p. 872-886, oct.-dec. 2014. Disponível em: <http://dx.doi.org/10.1590/S0103$65132013005000076>$. Acesso em: 14 out. 2015.

MU, J.; PENG, G.; MACLACHLAN, D. L. Effect of risk management strategy on NPD performance.

Technovation, [S.l.], v. 29, n. 3, p. 170-180, mar. 2009. Disponível em: <http://dx.doi.org/ 10.1016/j. technovation.2008.07.006>. Acesso em: 14 out. 2015.

MUNIZ, F. O. Características de empresas inovadoras: o projeto NUGI e suas empresas. Recife, 2013. $79 \mathrm{f}$. Dissertação (Mestrado em Engenharia de Produção) Universidade Federal de Pernambuco, 2013. Disponível em: <http://www.ppgep.org.br/dissertacoes/MA-0290. pdf $>$. Acesso em: 25 fev. 2015.

NAGANO, M. S.; STEFANOVITZ, J. P.; VICK, T. E. $\mathrm{O}$ contexto organizacional como aporte à inovação: um viés comparativo de casos em empresas brasileiras. Gestão \& Produção, São Carlos, SP, v. 21, n. 3, p. 477-490, jul.-set. 2014. Disponível em: < http:// dx.doi.org/10.1590/0104-530X510>. Acesso em: 25 fev. 2015.
O'CONNOR, G.; RAVICHANDRAN, T.; ROBESON, D. Risk management through learning: management practices for radical innovation success. Journal of High Technology Management Research, [S.l.], v. 19, n. 1, p. 70-82, sep. 2008. Disponível em: <http://dx.doi. org/10.1016/ j.hitech.2008.06.003 >. Acesso em: 14 out. 2015.

OEHMEN, J. et al. Analysis of the effect of risk management practices on the performance of new product development programs. Technovation, [S.I.], v. 34, n. 8, p. 441-453, aug. 2014. Disponível em: <http:// dx.doi.org/10.1016/j.technovation.2013.12.005>. Acesso em: 20 mar. 2015.

ORGANIZAÇÃO para a Cooperação e Desenvolvimento Econômico. Manual de Oslo: proposta de diretrizes para coleta e interpretação de dados sobre inovação tecnológica. 3. ed. Brasília, DF: FINEP, 2005. Disponível em: <http://www.oei.es/salactsi/oslo4.htm>. Acesso em: 14 fev. 2015.

RICE, M. P.; O'CONNOR, G. C.; PIERANTOZZI, $\mathrm{R}$. Implementing a learning plan to counter project uncertainty. Mit Sloan Management Review, USA, v. 49, n. 2, jan. 2008. Disponível em: < http://sloanreview. mit.edu/article/implementing-a-learning-plan-to-counterproject-uncertainty>. Acesso em: 14 out. 2015

ROVAI, R. L.; CATTINI, O.; PLONSKI, G. A. Gestão de riscos em projetos de inovação através da abordagem contingencial: análise conceitual e proposição de modelo estruturado para redução de incertezas em projetos complexos. Revista de Administração e Inovação, São Paulo, v. 10, n. 3, p. 269-295, jul.-set. 2013. Disponível em: <http://dx.doi.org/10.5773/rai.v10i3.1167>. Acesso em: 14 out. 2015.

ROZENFELD, H. et al. Gestão de desenvolvimento de produtos: uma referência para a melhoria do processo. São Paulo: Saraiva, 2006.

SALERNO, M. S. et al. Innovation processes: Which process for which project? Technovation, v. 35, n. 1 , p. 59-70, jan. 2015. Disponível em: < http://dx.doi. org/10.1016/j.technovation.2014.07 .012>. Acesso em: 2 nov. 2014. 
SANTOS, D. F. L. S. et al. Innovation efforts and performances of Brazilian firms. Journal of Business

Research, [S.I.], v. 67, n. 4, p. 527-535, apr.

2014. Disponível em: <http://dx.doi.org/10.1016/j

.jbusres.2013.11.009> . Acesso em: 14 out. 2015.

SILVA, D. O. da; BAGNO, R. B. S; SALERNO, M. S.

Modelos para a gestão da inovação: revisão e análise da literatura. Production, São Paulo, v. 24, n. 2, p. 477-490, apr. jun. 2014. Disponível em: <http://dx.doi. org/10.1590/S0103-65132013005000059>. Acesso em: 5 dez. 2014.

SPERO, J. et al. Research-on-research reports. Research Technology Management, [S.1.], v. 55, n. 6, p. 58-65, nov. 2012. Disponível em: <http://dx.doi.org/10.54 37/08956308X5506906 > . Acesso em: 7 out. 2015.

SRIVASTRAVA, S.; SINGH, D. Modifying stage-gate product development process with the application of customer perceived value components. International

Journal of Research in Management, Economics and Commerce, India, v. 2, n. 11, p. 106-126, nov. 2012. Disponível em: <http://www.indusedu.org/IJRMEC/ November2012(pdf)/12.pdf>. Acesso em: 16 nov. 2014.

STEFANOVITZ, J. P.; NAGANO, M. S. Gestão da inovação de produto: proposição de um modelo integrado. Production, São Paulo, v. 24, n. 2, p. 462476, apr.-jun. 2014. Disponível em: < http://dx.doi. org/10.1590/S0103-65132013005000062>. Acesso em: 14 out. 2015.

WANG, J. T.; LIN, W.; HUANG, Y. H. A performanceoriented risk management framework for innovative $R \& D$ projects. Technovation, [S. 1.], v. 30, n. 11, p. 601-611, nov. 2010. Disponível em: <http: //dx.doi.org/10.1016/j. technovation.2010.07.003 >. Acesso em: 5 jan. 2015.

ZWIKAEL, O.; AHN, M. The Effectiveness of Risk Management: An Analysis of Project Risk Planning Across Industries and Countries. Risk Analysis, [S.I.], v. 31, n. 1, p. 25-37, jan. 2011. Disponível em: < http//dx.doi.org/10.11 11/j.1539-6924.2010.01470.x> . Acesso em: 11 fev. 2015.

\footnotetext{
*Os autores agradecem ao IEL PE pela valiosa participação neste trabalho que contribuiu de forma decisiva para a construção da metodologia. Em especial, gostaríamos de agradecer aos analistas de inovação Rayanne Celly de Santana, Maria Celeste Maia, Viviam Renata Pessoa, Rodrigo de Matos, Evelyn Kruppa e Everaldo Vilela cujas colaborações permitiram a composição da ferramenta de análise. Agradecemos, também, aos avaliadores pelas recomendações sugeridas que, assim, ajudaram a aperfeiçoar este paper.
} 